\title{
Neural Network-based Adaptive Optimal Consensus Control of Leaderless Networked Mobile Robots
}

\author{
Haci Mehmet Guzey \\ Dept. of Elect. and Comp. Eng. \\ Missouri University of Sc. and \\ Tech. \\ Rolla, USA \\ hmgb79@mst.edu
}

\author{
$\mathrm{Hao} \mathrm{Xu}$ \\ Department of Elect. Eng. \\ College of Science and Engineering, \\ Texas A\&M - Corpus Christi, \\ Corpus Christi, USA \\ hao.xu@tamucc.edu
}

\author{
S. Jagannathan \\ Dept. of Elect. and Comp. Eng. \\ Missouri University of Sc. and \\ Tech. \\ Rolla, USA \\ saragp@mst.edu
}

\begin{abstract}
A novel neural network (NN)-based optimal adaptive consensus control scheme is introduced in this paper for networked mobile robots in the presence of unknown robot dynamics. Throughout the paper, two NNs are used. The unknown formation dynamics of each robot is identified by using the first $\mathrm{NN}$. The second $\mathrm{NN}$ is utilized to approximate a novel value function derived in this paper as a function of augmented error vector, which is comprised of the regulation and consensus-based formation errors of each robot. A novel near optimal controller is developed by using approximated value function and identified formation dynamics. The Lyapunov stability theorem is employed to derive the $\mathrm{NN}$ weight tuning laws and demonstrate the consensus achievement of the overall formation. The simulation results are depicted to show performance of our theoretical claims.
\end{abstract}

Keywords-Adaptive control; consensus; formation control; mobile robots; neural networks; optimal control; uncertain dynamics.

\section{INTRODUCTION}

Recently, the cooperative control of mobile robots has received increased attention [1], [2]. Motivated by possible applications such as surveillance or the formation control of satellites, several distributed controllers have been derived to enable agents to attain pre-specified tasks including consensus, coverage, and flocking or formation control. Given an initial position, the main task in formation control of mobile robots is to: i) drive the robot to a desired formation, and ii) maintain the desired formation while the network of robots move through the environment to reach their goal position and orientation.

Consensus-based techniques have been developed [3], [4] for formation control of agents. However, the past literature [1], [3]-[7] assumed that: i) the agent dynamics are known; ii) the optimal formation controller [5][6] is developed backward-in-time; and iii) linearized agent dynamics are taken for each robot/agent. When an agent is a mobile robot [7], the linearized robot dynamics are considered for consensus design. However, our previous work [8] has demonstarted that ignoring nonlinear dynamics may negatively affect the formation keeping of networked robots.

Therefore, forward-in-time optimal consensus-based formation control of networked mobile robots in the presence of unknown robot dynamics is still an open problem. Before developing the controller, the formation error dynamics and quadratic cost function of each robot are determined as a function of consensus based formation and regulation errors. It is found that the formation dynamics are nonlinear and can be expressed in affine form.

In the literature [9], [11], a variety of forward-in-time optimal controllers have been developed for agents described as a nonlinear affine systems with known or unknown dynamics. These online optimal controller approaches, also referred to as adaptive dynamics programming (ADP), require a significant number of iterations to learn the NN weights of optimal cost function approximation. However, inadequate iterations may cause instability of robot formation [9]. Therefore, a novel online ADP-based optimal controller was developed in [9] to relax the iterative approach. In this paper, a novel approach will be utilized to design a consensus-based optimal formation controller for networked mobile robots whose dynamics are considered uncertain without using iterations.

Two neural networks (NN) are employed in this ADPbased optimal controller design. The first NN is used for the identification of unknown formation dynamics of mobile robots while the second $\mathrm{NN}$ is utilized to approximate the value function, which becomes the solution to the HamiltonJacobi-Bellman (HJB) equation. In order to determine optimal consensus control of mobile robots, the approximated value function and approximated formation dynamics are utilized. Novel weight tuning laws are derived via Lyapunov stability method by minimizing formation keeping and regulation errors, and control torques of mobile robots.

The critical contributions of this work include: 1) the consensus-based optimal networked robot formation control in the presence of unknown nonlinear dynamics by using a novel value function estimation scheme; and 2) verification of the boundedness of the closed-loop robot dynamics and consensus achievement of the overall robot network.

The rest of the paper is constructed as follows. Section II gives a brief background on mobile robot dynamics, consensus based formation control and optimal regulation of continuous-time systems. The problem formulation is presented in Section III. Section IV considers the design of the optimal formation controller. Simulation results are revealed in Section V to verify our theoretical work before concluding with a summary of achievements in Section VI. 


\section{BACKGROUND AND PRELIMINARIES}

In this section, the dynamics of a single mobile robot is formulated as a nonlinear system in affine form, and the consensus based formation controller is addressed. A brief background is given on optimal regulation of continuous time systems.

\section{A. Mobile Robot Dynamics of Motion}

The dynamics of a single nonholonomic mobile robot [10] are a function of Cartesian positions and the bearing angle. The dynamics are subject to nonholonomic constraints given by

$\bar{M}\left(\bar{v}_{1}\right) \dot{\bar{v}}_{2}+\bar{C} \bar{v}_{2}+\bar{F}\left(\bar{v}_{2}\right)+\bar{G}\left(\bar{v}_{1}\right)+\tau_{d}=\bar{B}\left(\bar{v}_{1}\right) \tau-\bar{A}^{T} \Lambda$

With $\bar{v}_{1}=\left[\begin{array}{lll}x & y & \phi\end{array}\right]^{T} \in \mathbb{R}^{3 \times 1}$ and $\bar{v}_{2}=\left[\begin{array}{lll}\dot{x} & \dot{y} & \dot{\phi}\end{array}\right]^{T} \in \mathbb{R}^{3 \times 1}$ where $x, y$, and $\dot{x}, \dot{y}$ represent the Cartesian position and velocities, respectively, while $\phi$ and $\dot{\phi}$ denote bearing angles and angular velocity, respectively. The matrices, $\bar{M}(\bullet) \in \mathbb{R}^{3 \times 3}$

$, \bar{B} \in \mathbb{R}^{3 \times 2}, \bar{C} \in \mathbb{R}^{3 \times 3}$, and $\bar{A} \in \mathbb{R}^{3 \times 2}$ represent inertial, input transformation, Coriolis and constraint matrices respectively. The vectors $\tau \in \mathbb{R}^{2 \times 1}, \tau_{d} \in \mathbb{R}^{3 \times 1}, \Lambda \in \mathbb{R}^{2 \times 1}, \bar{G}(\bullet) \in \mathbb{R}^{3 \times 1}$ and $\bar{F}(\bullet) \in \mathbb{R}^{3 \times 1}$ denote the control torque, bounded disturbance, the constraint forces, gravitational vector and friction vectors, respectively[10].

By using the fact that inertia matrix, $\bar{M}\left(\bar{v}_{1}\right)$, is invertible [3], equation (1) is rewritten as

$$
\dot{\bar{v}}_{2}=\bar{M}\left(\bar{v}_{1}\right)^{-1}\left(\bar{C}_{2}-\bar{F}\left(\bar{v}_{2}\right)-\bar{G}\left(\bar{v}_{1}\right)-\tau_{d}-\bar{A}^{T} \Lambda\right)+\bar{M}\left(\bar{v}_{1}\right)^{-1} \bar{B}\left(\bar{v}_{1}\right) \tau .
$$

The state space representation of (2) can be given by

$\dot{\bar{v}}_{1}=\bar{v}_{2}$

$\dot{\bar{v}}_{2}=\bar{f}(\bar{v})+\bar{g}(\bar{v}) \tau$

where $\bar{v}=\left[\begin{array}{ll}\bar{v}_{1}^{T} & \bar{v}_{2}^{T}\end{array}\right]^{T} \in \mathbb{R}^{6 \times 1}$,

$\bar{f}\left(\bar{v}_{2}, \bar{v}_{1}\right)=\bar{M}\left(\bar{v}_{1}\right)^{-1}\left(\bar{C} \bar{v}_{2}-\bar{F}\left(\bar{v}_{2}\right)-\bar{G}\left(\bar{v}_{1}\right)-\tau_{d}-\bar{A}^{T} \Lambda\right) \in \mathbb{R}^{3 \times 1}$ and $\bar{g}\left(\bar{v}_{1}\right)=\bar{M}\left(\bar{v}_{1}\right)^{-1} \bar{B}\left(\bar{v}_{1}\right) \in \mathbb{R}^{3 \times 2}$ denote the nonlinear functions acting as the system dynamics. Alternative to (3), the state space representation can be expressed in nonlinear affine form as

$\dot{\bar{v}}=\bar{f}_{r}(\bar{v})+\bar{g}_{r}(\bar{v}) \tau$,

where $\bar{f}_{r}(\bar{v})=\left[\begin{array}{ll}\bar{v}_{2}^{T} & \bar{f}(\bar{v})^{T}\end{array}\right]^{T} \in \mathbb{R}^{6 \times 1}$ and ,

$\bar{g}_{r}(\bar{v})=\left[\begin{array}{ll}0_{2 \times 3} & \bar{g}(\bar{v})^{T}\end{array}\right]^{T} \in \mathbb{R}^{6 \times 2} \quad$ represents the internal dynamics and control coefficient matrix, respectively. In a formation control problem with $n$ mobile robots involved, the dynamics of the $i^{\text {th }}$ robot can be written as

$\dot{\bar{v}}_{i}=\bar{f}_{r, i}\left(\bar{v}_{i}\right)+\bar{g}_{r, i}\left(\bar{v}_{i}\right) \tau_{i} \forall i=1,2, \cdots, n$.

Here, all the robots in the network are assumed to have dynamics similar to the one given in (5) for $i^{\text {th }}$ robot. The following assumption is needed before we proceed:
Assumption 1: The state vector, $\bar{v}_{i}$, in (5) is available and the input transformation matrix satisfies $\left\|\bar{g}_{r, i}\left(\bar{v}_{i}\right)\right\| \leq \bar{g}_{\max } \forall i=1,2, \ldots, n$.

The system (5) is considered controllable and without loss of generality, this implies that a continuous control policy can be found that stabilizes the robot dynamics in (5), where $\bar{v}_{i}=0$ is a unique equilibrium point on a set $\Omega \subseteq \mathbb{R}^{6}$. Recall that the main objective of formation control is to reach a desired state, $\bar{v}_{i, d} \in \mathbb{R}^{6 \times 1}$, for the $i^{\text {th }}$ mobile robot while maintaining the formation intact.

Next, the regulation error is defined as the difference between actual state, $\bar{v}_{i}$ and its desired value, $\bar{v}_{i, d}$ as

$\tilde{v}_{i}=\bar{v}_{i}-\bar{v}_{i, d}$

with $\tilde{v}_{i} \in \mathbb{R}^{6 \times 1}$ being the regulation error of the $i^{\text {th }}$ robot. Since the desired positions and desired velocities are held for regulation, $\dot{\bar{v}}_{i, d}=0$, hence, the mobile robot regulation error dynamics from (6) can be represented as

$\dot{\tilde{v}}_{i}=\dot{\bar{v}}_{i}-\dot{\bar{v}}_{i, d}=\dot{\bar{v}}_{i}=\bar{f}_{r, i}\left(\bar{v}_{i}\right)+\bar{g}_{r, i}\left(\bar{v}_{i}\right) \tau_{i}$.

Further, the regulation error dynamics (7) can be represented as

$\dot{\tilde{v}}_{i}=f_{r, i}\left(\tilde{v}_{i}\right)+g_{r, i}\left(\tilde{v}_{i}\right) \tau_{i}$

where $f_{r, i}\left(\tilde{v}_{i}\right)=\bar{f}_{r, i}\left(\bar{v}_{i}\right)=\bar{f}_{r, i}\left(\tilde{v}_{i}+\bar{v}_{i, d}\right)$ and

$g_{r, i}\left(\tilde{v}_{i}\right)=\bar{g}_{r, i}\left(\bar{v}_{i}\right)=\bar{g}_{r, i}\left(\tilde{v}_{i}+\bar{v}_{i, d}\right)$.

Assumption 1 states that $\left\|g_{r, i}\left(\tilde{v}_{i}\right)\right\| \leq g_{\max } \forall i=1,2, \ldots, n$. Additionally in a formation controller design, consensus has to be attained to maintain formation which is discussed next.

\section{B. Consensus-based Formation Controller}

In this subsection, the classical consensus-based controller design is discussed briefly. The following assumption on communication aspects of the robots within formation is needed before introducing the consensus methodology.

Assumption 2 [5]: The connectivity graph of the mobile robot network will be undirected as well as connected.

This assumption is utilized to contruct the formation dynamics of each robot since the formation dynamics are a function of neighbor robots. Next, the main goal of robot formation control is reaching consensus by controlling the robot formation error defined as

$\delta_{i}=\sum_{j=1}^{n}\left[\left(\tilde{v}_{i}-\tilde{v}_{j}\right)\right] \in \mathbb{R}^{6 \times 1}, j \in N_{i}$

with $\tilde{v}_{i}$ being the regulation error of the $i^{t h}$ robot and $N_{i}$ is the set of robots in the neighborhood of the $i^{\text {th }}$ robot. In order to design an optimal consensus based formation controller for nonlinear systems, both the regulation and formation errors need to be considered. Next, classical 
optimal control background is presented before introducing our proposed controller.

\section{Optimal Regulation of Continuous time systems}

Consider the nonlinear mobile robot regulation errors dynamics (8) in nonlinear affine form. The goal here is to derive an optimal control policy by minimizing the value function

$$
V_{i}\left(\tilde{v}_{i}\right)=\int_{t}^{\infty}\left(Q\left(\tilde{v}_{i}(\vartheta)\right)+\tau_{i}^{T}(\vartheta) R \tau_{i}(\vartheta)\right) d \vartheta
$$

with $Q\left(\tilde{v}_{i}(\vartheta)\right) \geq 0 \in \mathbb{R}$ being a positive semi-definite function to penalize the regulation error while $R \in \mathbb{R}^{2 \times 2}$ is a positive-definite matrix to penalize the control input of the $i^{\text {th }}$ robot. For the sake of simplicity in notation, the penalizing factor, $Q\left(\tilde{v}_{i}(\vartheta)\right)$, is presented as $Q\left(\tilde{v}_{i}\right)$ from now.

Next, define the Hamiltonian as

$$
H\left(\tilde{v}_{i}, \tau_{i}\right)=Q\left(\tilde{v}_{i}\right)+\tau_{i}^{T} R \tau_{i}+V_{\tilde{v}_{i}}^{T}\left[f_{r, i}\left(\tilde{v}_{i}\right)+g_{r, i}\left(\tilde{v}_{i}\right) \tau_{i}\right]
$$

with $V_{\tilde{v}_{i}}=\frac{\partial V_{i}\left(\tilde{v}_{i}, t\right)}{\partial \tilde{v}_{i}}$ being the gradient of the value function $V_{i}\left(\tilde{v}_{i}\right)$. The optimal controller scheme [9] is obtained by using the stationarity condition $\partial H\left(\tilde{v}_{i}, \tau_{i}\right) / \partial \tau_{i}=0$, which yields

$\tau_{i}^{*}\left(\tilde{v}_{i}\right)=-\frac{1}{2} R^{-1} g_{r, i}^{T}\left(\tilde{v}_{i}\right) V_{\tilde{v}_{i}}^{*}\left(\tilde{v}_{i}\right)$.

Replacing (12) into (11) yields the time-varying HJB equation as

$$
Q\left(\tilde{v}_{i}\right)+V_{\tilde{v}_{i}}^{* T} f_{r, i}\left(\tilde{v}_{i}\right)-V_{\tilde{v}_{i}}^{* T} g_{r, i}\left(\tilde{v}_{i}\right) R^{-1} g_{r, i}{ }^{T}\left(\tilde{v}_{i}\right) V_{\bar{v}_{i}}^{*} / 4=0
$$

where $V_{\tilde{v}_{i}}^{*}$ represents the derivative of optimal time-varying value function of the $i^{\text {th }}$ robot. The value function which is the solution to the HJB equation is utilized to obtain the optimal controller. On the other hand, obtaining an analytical closed-form solution of the HJB equation (13) is extremely difficult. Hence, an approximation based technique-so called ADP is used to approximate the solution of (13) online.

Lemma 1[9]: Consider the regulation errors dynamics of the $i^{t h}$ robot (8) with the value function (10) and the optimal controller (12). Let $J\left(\tilde{v}_{i}\right)$ be a continuously differentiable, radially unbounded Lyapunov candidate such that $\dot{J}\left(\tilde{v}_{i}\right)=J_{\tilde{v}_{i}}^{\mathrm{T}}\left(\tilde{v}_{i}\right) \dot{\tilde{v}}_{i}=J_{\tilde{v}_{i}}^{\mathrm{T}}\left(\tilde{v}_{i}\right)\left(f\left(\tilde{v}_{i}\right)+g\left(\tilde{v}_{i}\right) \tau_{i}^{*}\right)<0$ where $J_{\tilde{v}_{i}}\left(\tilde{v}_{i}\right)$ is the partial derivative of the Lyapunov candidate, $J\left(\tilde{v}_{i}\right)$, with respect to regulation error, $\tilde{v}_{i}$. In addition, let $\bar{Q}\left(\tilde{v}_{i}\right) \in \mathbb{R}$ be a positive-definite matrix, i.e., $\forall \tilde{v}_{i} \neq 0, \tilde{v}_{i} \in \Omega$, $\left\|\bar{Q}\left(\tilde{v}_{i}\right)\right\|>0, \quad$ and $\quad \tilde{v}_{i}=0 \Rightarrow\left\|\bar{Q}\left(\tilde{v}_{i}\right)\right\|=0, \quad$ and $\bar{Q}_{\min } \leq\left\|\bar{Q}\left(\tilde{v}_{i}\right)\right\| \leq \bar{Q}_{\max } . \quad$ Further, let $\quad \bar{Q}\left(\tilde{v}_{i}\right) \quad$ satisfies $\lim _{\tilde{v}_{i} \rightarrow \infty} \bar{Q}\left(\tilde{v}_{i}\right)=\infty$ as well as

$V_{\tilde{v}_{i}}^{* \mathrm{~T}} \bar{Q}\left(\tilde{v}_{i}\right) J_{\tilde{v}_{i}}=r\left(\tilde{v}_{i}, \tau_{i}^{*}\right)=Q\left(\tilde{v}_{i}\right)+\tau_{i}^{* \mathrm{~T}} R \tau_{i}^{*}$.

where $r\left(\tilde{v}_{i}, \tau_{i}^{*}\right)$ being the cost to go function. Then, the following relation will hold

$$
J_{\tilde{v}_{i}}^{\mathrm{T}}\left(f\left(\tilde{v}_{i}\right)+g\left(\tilde{v}_{i}\right) \tau_{i}^{*}\right)=-J_{\tilde{v}_{i}}^{\mathrm{T}} \bar{Q}\left(\tilde{v}_{i}\right) J_{\tilde{v}_{i}} .
$$

In a consensus-based control, as the complete formation dynamics are functions of both the regulation and the formation errors, a novel cost function had to be defined. In the next section, a problem statement of the consensus-based optimal formation controller is formulated.

\section{PROBLEM FORMULATION}

The augmented formation dynamics are determined, and the near optimal consensus-based formation controller of mobile robots is derived by using ADP in this section.

\section{A. Formation Dynamics of Mobile Robots}

The formation dynamics are established by augmenting the regulation and formation errors together. The dynamics of the formation errors (9) can be derived as

$\dot{\delta}_{i}=\sum_{j=1}^{n}\left(f_{r, i}\left(\tilde{v}_{i}\right)+g_{r, i}\left(\tilde{v}_{i}\right) \tau_{i}-f_{r, j}\left(\tilde{v}_{j}\right)-g_{r, j}\left(\tilde{v}_{j}\right) \tau_{j}\right), j \in N_{i}(15$

Now, combining regulation and formation errors of the $i^{\text {th }}$ robot, $\zeta_{i}=\left[\begin{array}{ll}\tilde{v}_{i}^{T} & \delta_{i}^{T}\end{array}\right]^{T} \in \mathbb{R}^{12 \times 1}$, and utilizing (8) and (15) yields the formation error dynamics of the $i^{\text {th }}$ robot as

$$
\dot{\zeta}_{i}=\left[\begin{array}{c}
f_{r, i}\left(\tilde{v}_{i}\right)+g_{r, i}\left(\tilde{v}_{i}\right) \tau_{i} \\
\sum_{j=1}^{n}\left(f_{r, i}\left(\tilde{v}_{i}\right)+g_{r, i}\left(\tilde{v}_{i}\right) \tau_{i}-f_{r, j}\left(\tilde{v}_{j}\right)-g_{r, j}\left(\tilde{v}_{j}\right) \tau_{j}\right)
\end{array}\right], j \in N_{i} .
$$

In the nonlinear affine form, (16) is represented as

$$
\dot{\zeta}_{i}=\left[\sum_{j=1}^{n}\left(f_{r, i}\left(\tilde{v}_{i}\right)-f_{r, j}\left(\tilde{v}_{j}\right)-g_{r, j}\left(\tilde{v}_{j}\right) \tau_{j}\right)\right]+\left[\begin{array}{c}
g_{r, i}\left(\tilde{v}_{i}\right) \\
n g_{r, i}\left(\tilde{v}_{i}\right)
\end{array}\right] \tau_{i} j \in N_{i} .
$$

The formation error dynamics of $i^{\text {th }}$ mobile robot (17) can be represented as

$\dot{\zeta}_{i}=f_{i}\left(\tilde{v}_{N i}, \tau_{N i}\right)+g_{i}\left(\tilde{v}_{i}\right) \tau_{i}$

where $\tau_{j} \in \tau_{N i}, \forall j \in N_{i}$ and $\tilde{v}_{j} \in \tilde{v}_{N i}, \forall j \in N_{i}$ represents the control input and regulation error vectors of all the robots in the neighborhood of the $i^{t h}$ robot, respectively, with $\tilde{v}_{N i}$ being the set of regulation errors of robots in the neighborhood of $i^{t h}$ robot and

$$
\begin{aligned}
& f_{i}\left(\tilde{v}_{N i}, \tau_{N i}\right)=\left[\begin{array}{c}
f_{r, i}\left(\tilde{v}_{i}\right) \\
\sum_{j=1}^{n}\left(f_{r, i}\left(\tilde{v}_{i}\right)-f_{r, j}\left(\tilde{v}_{j}\right)-g_{r, j}\left(\tilde{v}_{j}\right) \tau_{j}\right)
\end{array}\right] \in \mathbb{R}^{12 \times 1}, \\
& g_{i}\left(\tilde{v}_{i}\right)=\left[\begin{array}{c}
g_{r, i}\left(\tilde{v}_{i}\right) \\
n g_{r, i}\left(\tilde{v}_{i}\right)
\end{array}\right] \in \mathbb{R}^{12 \times 1} .
\end{aligned}
$$

Remark 3: In [13], the formation dynamics of each robot are functions of its own leader only; however, the formation dynamics in the consensus approach (18) is a function of robots in the neighborhood. This aspect essentially provides robustness against communication failures. The controller torque, $\tau_{i}$, in augmented errors dynamics (18) of the $i^{\text {th }}$ robot is formulated in a decentralized way. These formation 
dynamics are not known beforehand as they are a function of regulation errors and control inputs of other robots in the network. Therefore, the unknown formation dynamics (18) are identified through an NN identifier which is subsequently utilized in the optimal formation controller design. First, a consensus based value function is defined.

\section{B. Consensus-based Value Function}

In order to derive the consensus-based optimal formation controller, a novel value function is proposed for the $i^{\text {th }}$ robot as

$V_{i}\left(\zeta_{i}\right)=\int_{t}^{\infty}\left(Q\left(\zeta_{i}(\vartheta)\right)+\tau_{i}^{T}(\vartheta) R \tau_{i}(\vartheta)\right) d \vartheta$

with $Q\left(\zeta_{i}\right)=\chi_{1} \tilde{v}_{i}^{T} \Pi_{i} \tilde{v}_{i}+\chi_{2} \delta_{i}^{T} \Pi_{i} \delta_{i} \quad$ where $\quad \Pi_{i} \in \mathfrak{R}^{6 \times 6}$ represents a positive definite matrix to penalize regulation and formation errors, respectively. Additionally, $\chi_{1}, \chi_{2}$ are positive design constants, and $R$ is a positive-definite constant matrix for each mobile robot.

The value function (19), along with augmented error $\zeta_{i}$, as its input, can be represented by using a $\mathrm{NN}$ with activation function on a compact set, $\Omega$, in the form [11] as

$V_{i}\left(\zeta_{i}\right)=\theta_{V_{i}}^{T} \psi_{V i}\left(\zeta_{i}\right)+\varepsilon_{V_{i}}\left(\zeta_{i}\right)$

with $\theta_{V i} \in \mathbb{R}^{L \times 1}$ being the target NN weight vector where $L$ is the number of neurons, $\psi_{V_{i}}\left(\zeta_{i}\right): \mathbb{R}^{12} \times[0, \infty) \rightarrow \mathbb{R}^{L}$ is a bounded activation function of regulation errors and formation errors of each mobile robot and $\varepsilon_{V_{i}}\left(\zeta_{i}\right)$ is the NN reconstruction error.

The target $\mathrm{NN}$ weights, $\theta_{V_{i}}$, and reconstruction error, $\varepsilon_{V_{i}}\left(\zeta_{i}\right)$, are both assumed to be bounded above such that $\left\|\theta_{V_{i}}\right\| \leq \theta_{V M_{i}}$ and $\left\|\varepsilon_{V_{i}}\left(\zeta_{i}\right)\right\| \leq \varepsilon_{M i}$, with $\theta_{V M_{i}}$ and $\varepsilon_{M i}$ are positive constants [9]. Additionally, the gradient of the NN reconstruction error with respect to $\zeta_{i}$ is assumed to be bounded above, such that $\left\|\nabla_{\xi_{i}} \varepsilon_{V i}\left(\zeta_{i}\right)\right\| \leq \varepsilon_{V M i}$ [9], with $\varepsilon_{V M i}$ is a positive constant as well. In the next section, an adaptive optimal NN-based formation controller scheme is presented.

\section{Optimal Adaptive Consensus Controller Design}

The optimal adaptive consensus-based formation controller is derived for mobile robots in the presence of uncertain robot formation dynamics. First, the unknown formation dynamics are identified through a NN. Second, a novel NN-based adaptive optimal consensus controller is developed. Further, the optimality and consensus ability of the overall robot network are stated in Theorem 2 based on the formation controller defined for each mobile robot individually. The identification of $i^{\text {th }}$ robot formation dynamics is introduced next.

\section{A. NN-based Identifier}

Consider the formation error dynamics of ith robot (18) in nonlinear affine form. On a compact set, $\Omega$, the mobile robot formation error dynamics can be represented as [12]
$f_{i}\left(\tilde{v}_{N i}, \tau_{N i}\right)=\theta_{f i}^{T} \psi_{f i}\left(\tilde{v}_{N i}, \tau_{N i}\right)+\varepsilon_{f i}$,

$g_{i}\left(\tilde{v}_{i}\right)=\theta_{g i}^{T} \psi_{g i}\left(\tilde{v}_{i}\right)+\varepsilon_{g i}$

with $\theta_{f i} \in \mathbb{R}^{l \times 6}, \quad \theta_{g i} \in \mathbb{R}^{l \times 6}$ being $\mathrm{NN}$ desired weight matrices where $L$ is the number of neurons, $\psi_{f i}: \mathbb{R}^{6} \rightarrow \mathbb{R}^{L}$, and $\psi_{g i}: \mathbb{R}^{6 \times 2} \rightarrow \mathbb{R}^{L \times 2}$ are activation functions, and $\mathcal{E}_{f i} \in \mathbb{R}^{6 \times 1}, \mathcal{E}_{g i} \in \mathbb{R}^{6 \times 2}$ are $\mathrm{NN}$ reconstruction errors. Even though the dynamics of the neighbor robots are uncertain to the $i^{\text {th }}$ robot, the regulation errors and control torques are transmitted to identify formation error dynamics by using the connectivity graph.

Then, the robot formation dynamics (8) can be expressed by using (21) as

$\dot{\zeta}_{i}=f_{i}\left(\tilde{v}_{N i}, \tau_{N i}\right)+g_{i}\left(\tilde{v}_{i}\right) \tau_{i}$

$=\theta_{f i}^{T} \psi_{f i}\left(\tilde{v}_{N i}, \tau_{N i}\right)+\theta_{g i}^{T} \psi_{g i}\left(\tilde{v}_{i}\right) \tau_{i}+\varepsilon_{f i}+\varepsilon_{g i} \tau_{i}$

$=\left[\begin{array}{c}\theta_{f i} \\ \theta_{g i}\end{array}\right]^{T}\left[\begin{array}{cc}\psi_{f i}\left(\tilde{v}_{N i}, \tau\right) & 0 \\ 0 & \psi_{g i}\left(\tilde{v}_{i}\right)\end{array}\right]\left[\begin{array}{c}1 \\ \tau_{i}\end{array}\right]+\varepsilon_{f i}+\varepsilon_{g i} \tau_{i}$

$=\theta_{I i}^{T} \psi_{I i}\left(\tilde{v}_{N i}, \tau_{N i}\right) \bar{\tau}_{i}+\varepsilon_{I i}$

with $\theta_{l i}=\left[\begin{array}{lll}\theta_{f i}^{T} & \theta_{g i}^{T}\end{array}\right]^{T} \in \mathbb{R}^{2 l \times 6}$ and $\psi_{I i}\left(\xi_{i}\right)=\operatorname{diag}\left\{\psi_{f i}\left(\tilde{v}_{N i}, \tau\right), \psi_{g i}\left(\tilde{v}_{i}\right)\right\}$, $\psi_{I i}: \mathbb{R}^{6 \times 3} \rightarrow \mathbb{R}^{2 l \times 3}$, which represent the $\mathrm{NN}$ identifier desired weights matrix and basis function, respectively, where $\bar{\tau}_{i}=\left[\begin{array}{ll}1 & \tau_{i}^{T}\end{array}\right]^{T} \in \mathbb{R}^{3}$ and $\varepsilon_{l i}=\varepsilon_{f i}+\varepsilon_{g i} \tau_{i}$ denote the augment control input vector and the $\mathrm{NN}$ identifier reconstruction error, respectively. Since $\psi_{I i}(\tilde{v})$ is known and $\theta_{I i}^{T}$ is unknown, the dynamics (22) can be estimated as

$\dot{\hat{\xi}}_{i}=\hat{\theta}_{I i}^{T} \psi_{I i}\left(\tilde{v}_{N i}, \tau_{N i}\right) \bar{\tau}_{i}+K e_{i}$

where $\hat{\theta}_{I i} \in \mathbb{R}^{2 l \times 6}$ is the estimation of the NN weight matrix, $K$ is a design parameter that is utilized to stabilize the NN identifier, and $e_{i}=\xi_{i}-\hat{\xi}_{i}$ being the state estimation error vector.

By substituting (23) with (22), the state estimation error dynamics of $i^{\text {th }}$ robot can be given by

$\dot{e}_{i}=\dot{\xi}_{i}-\dot{\hat{\xi}}_{i}=\tilde{\theta}_{I i}^{T} \psi_{I i}\left(\tilde{v}_{N i}, \tau_{N i}\right) \bar{\tau}_{i}+\varepsilon_{I i}-K e_{i}$

with $\tilde{\theta}_{I i}=\left(\theta_{I i}-\hat{\theta}_{I i}\right) \in \mathbb{R}^{2 l \times 6}$ being the $\mathrm{NN}$ weight estimation error matrix. The tuning law for $\hat{\theta}_{I i}$ is derived to force the actual NN weights matrix close to desired NN weights as

$\dot{\hat{\theta}}_{I i}=-\lambda_{I i} \hat{\theta}_{I i}+\psi_{I i}\left(\tilde{v}_{N i}, \tau_{N i}\right) \bar{\tau}_{i} e_{i}$

where $\lambda_{I i}$ is the adaptation parameter of the $\mathrm{NN}$ identifier, which satisfies $\lambda_{I i}>0$. Since $\dot{\tilde{\theta}}_{I i}=-\dot{\hat{\theta}}_{I i}$, and by utilizing (25) , the $\mathrm{NN}$ identifier weights estimation error dynamics can be given by

$\dot{\tilde{\theta}}_{I i}=\lambda_{I i} \hat{\theta}_{I i}-\psi_{I i}\left(\tilde{v}_{N i}, \tau_{N i}\right) \bar{\tau}_{i} e_{i}$

The NN identifier (23) is utilized to derive the optimal controller for the mobile robot formation as introduced in the next section. 


\section{B. Optimal Consensus Controller Design}

In order to estimate the value function, $V_{i}\left(\zeta_{i}\right)$ of $i^{\text {th }}$ robot, define

$\hat{V}_{i}\left(\zeta_{i}\right)=\hat{\theta}_{V_{i}}^{T} \psi_{V_{i}}\left(\zeta_{i}\right)$

where $\hat{V}_{i}\left(\zeta_{i}\right)$ is the approximation of the value function, and $\hat{\theta}_{V_{i}} \in \mathfrak{R}^{L}$ is the actual NN weights for the value function. With the NN approximation of value function (27), the approximated Hamiltonian is given by $\hat{H}_{i}\left(\zeta_{i}, \tau_{i}\right)=\hat{\theta}_{V_{i}}^{T} \nabla_{t} \psi_{V_{i}}\left(\zeta_{i}\right)+\hat{\theta}_{V_{i}}^{T} \nabla_{\xi_{i}} \psi_{V_{i}}\left(\zeta_{i}\right) \hat{f}_{i}\left(\zeta_{i}\right)$

$-\frac{1}{4} \hat{\theta}_{V_{i}}^{T} \nabla_{\xi_{i}} \psi_{V_{i}}\left(\zeta_{i}\right) \hat{D}\left(\tilde{v}_{i}\right) \nabla_{\xi_{i}}^{T} \psi_{V_{i}}\left(\zeta_{i}\right) \hat{\theta}_{V_{i}}$

where $\hat{D}\left(\tilde{v}_{i}\right)=\hat{g}_{i}^{T}\left(\tilde{v}_{i}\right) R^{-1} \hat{g}_{i}\left(\tilde{v}_{i}\right)$ is derived from the $\mathrm{NN}$ identifier. The estimated controller torque is given by $\hat{\tau}_{i}\left(\zeta_{i}\right)=-\frac{1}{2} R^{-1} \hat{g}_{i}^{T}\left(\tilde{v}_{i}\right) \nabla_{\xi_{i}} \psi_{V_{i}}^{T}\left(\zeta_{i}\right) \hat{\theta}_{V_{i}}$,

which is obtained as a function of approximated formation dynamics. The objective here is to obtain the Hamiltonian estimation (23) along the robot trajectory, such that the optimality in terms of controller effort can be achieved. Hence, the NN tuning law is found by minimizing (26) as well as stabilizing the formation dynamics of $i^{\text {th }}$ robot. By using normalized gradient descent, the NN tuning law can be given

$\dot{\hat{\theta}}_{V_{i}}=-\gamma_{1} \frac{\hat{\eta}}{\left(1+\hat{\eta}^{T} \hat{\eta}\right)^{2}} \hat{H}_{i}\left(\zeta_{i}, \tau_{i}\right)+\gamma_{2} \nabla_{\xi_{i}} \psi_{V_{i}}\left(\zeta_{i}\right) \hat{D}\left(\tilde{v}_{i}\right) J_{\zeta_{i}}\left(\zeta_{i}\right) / 2$

with $\hat{\eta}=\nabla_{\xi_{i}} \psi_{V_{i}}\left(\zeta_{i}\right) \hat{f}_{i}\left(\zeta_{i}\right)-\frac{1}{2} \nabla_{\xi_{i}} \psi_{V_{i}}\left(\zeta_{i}\right) \hat{D}\left(\tilde{v}_{i}\right) \nabla_{\zeta_{i}}^{T} \psi_{V_{i}}\left(\zeta_{i}\right) \hat{\theta}_{V_{i}}, \gamma_{1}$, $\gamma_{2}$ are positive design parameters and $J_{\zeta_{i}}\left(\zeta_{i}\right)$ is partial derivative of the Lyapunov candidate, $J\left(\zeta_{i}\right)$, with respect to $\zeta_{i}$. Note that, the definition of Lyapunov candidate, $J\left(\zeta_{i}\right)$ is similar to Lemma 1 . The second term in (30) ensures that the formation and regulation errors remain bounded while the NN weights learn the optimal value function [9].

In Fig. 1, our proposed controller is drawn as a flowchart. The desired positions, heading angle, and velocities are utilized to generate regulation error of each robot. The regulation errors and the torques of neighbor robots are employed to obtain formation dynamics which is subsequently utilized to generate controller torque of $i^{\text {th }}$ robot.

Theorem 1: Consider the formation error dynamics of the $i^{\text {th }}$ robot (18) in a networked group. Assume that each robot broadcasts its own regulation errors and control inputs (torques) over the network with no communication delays. Let the communication graph of the robot network satisfies Assumptions 1 and 2. Let the NN weight tuning law for the identifier and value functions be presented as (25), and (30), respectively, and the approximated control input given by (29). Then, there exists positive design constants, $\gamma_{1}>0$, $\gamma_{2}>0$, such that the formation $\left\|\delta_{i}\right\|$ and the regulations errors $\left\|\tilde{v}_{i}\right\|$ are bounded. In addition, the identification error $\left\|e_{i}\right\|$ and $\mathrm{NN}$ weights estimation errors of the $\mathrm{NN}$ identifier, $\left\|\tilde{\theta}_{I i}\right\|$ and the value function, $\left\|\tilde{\theta}_{V i}\right\|$ are all bounded. Moreover, the bounds are a function of initial regulation and formation errors, i.e., bounds $B_{J_{\zeta_{i}}, 0}$, initial identification errors bound $B_{e_{i}, 0}$ and initial weight estimation errors for $\mathrm{NN}$ identifier and value function $B_{\theta_{I i}, 0}, B_{\theta_{V i}, 0}$, respectively.

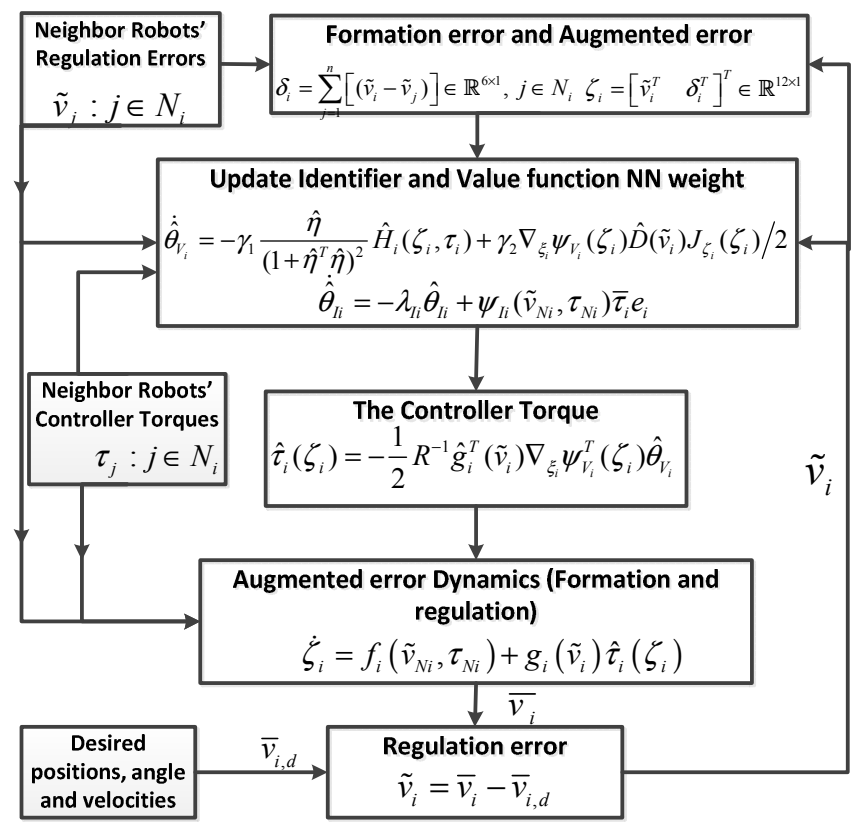

Fig. 1. Flowchart of the controller scheme.

Theorem 2: Consider the formation error dynamics (18) of $i^{\text {th }}$ robot in a leaderless networked mobile robots group based on their neighboring sets. Let the $\mathrm{NN}$ weights tuning laws of the identifier and the value function estimation be given by (25), and (30), respectively. Then, the controller torque given by (29) of each mobile robot minimizes the value functions (19) and also guarantees that the robots can reach consensus over their regulation errors. Moreover, the leaderless group of networked robots move toward their desired position while maintaining the consensus and, eventually attain near the goal position of the group.

\section{Simulation Results}

The performance of an adaptive optimal consensus-based controller (29) for mobile robot formation is demonstrated in this section. Four mobile robots are employed in the network. The initial positions and velocities of each robot are chosen as

$$
\begin{aligned}
& x_{1}(0)=0, x_{2}(0)=20, x_{3}(0)=0, x_{4}(0)=-20, \\
& y_{1}(0)=20, y_{2}(0)=0, y_{3}(0)=-20, y_{4}(0)=0, \\
& \dot{x}_{1}(0)=\dot{x}_{2}(0)=\dot{x}_{3}(0)=\dot{x}_{4}(0)=\dot{y}_{1}(0)=\dot{y}_{2}(0)=\dot{y}_{3}(0)=\dot{y}_{4}(0)=0 .
\end{aligned}
$$


Each robot is controlled through an adaptive optimal formation controller (29) and forced to reach its goal position and velocity by consuming minimum energy. Desired locations were chosen as

$x_{1 d}=-14, x_{2 d}=-8, x_{3 d}=-14, x_{4 d}=-22, y_{1 d}=21, y_{2 d}=15$,

$y_{3 d}=9, y_{4 d}=15$ and the desired Cartesian velocities were all set to zero. Second, the basis vector of value function estimation is defined as

$\left[\zeta_{1}^{2}, \cdots, \zeta_{12}^{2}, \zeta_{1}^{2} \zeta_{2}, \ldots, \zeta_{12}^{2} \zeta_{1}, \zeta_{1}^{3} \zeta_{2}^{2}, \ldots, \zeta_{12}^{3} \zeta_{1}^{2}, \zeta_{1}^{4} \zeta_{2}^{3}, \ldots, \zeta_{12}^{4} \zeta_{1}^{3}, \zeta_{1}^{6}, \zeta_{2}^{6}\right]^{T} \in \mathfrak{R}^{50 \times 1}$ with $\left[\zeta_{1}, \cdots, \zeta_{6}\right]$ being regulation error, and $\left[\zeta_{7}, \cdots, \zeta_{12}\right]$ being the formation error of $i^{\text {th }}$ robot. The activation function of the identifier $\mathrm{NN}$ is also selected in the same manner. The initial NN weights are chosen as small random numbers, $W_{I 0} \in 0.01 * \operatorname{rand}(50,1)$, for both identifier and value function NN. Fig. 2 illustrates the evaluation of the neural network weights during the online learning of value function.
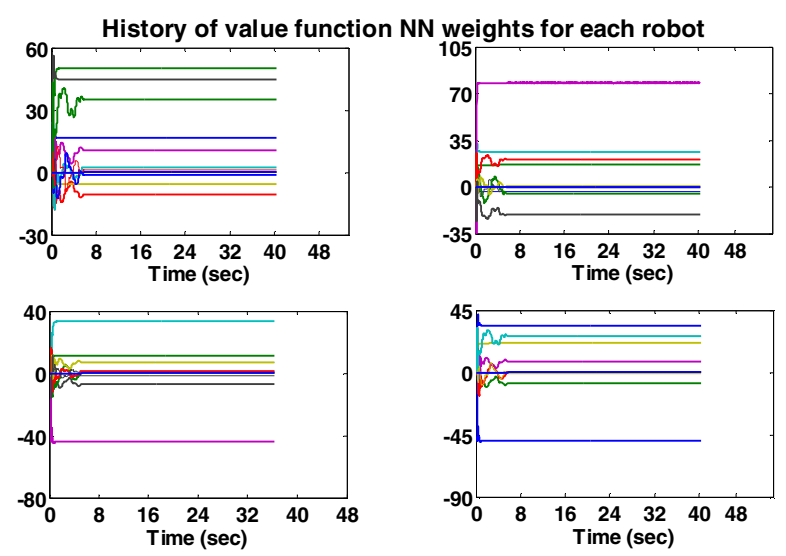

Fig. 2. Evaluation of Value fucntion $\mathrm{NN}$ weights.
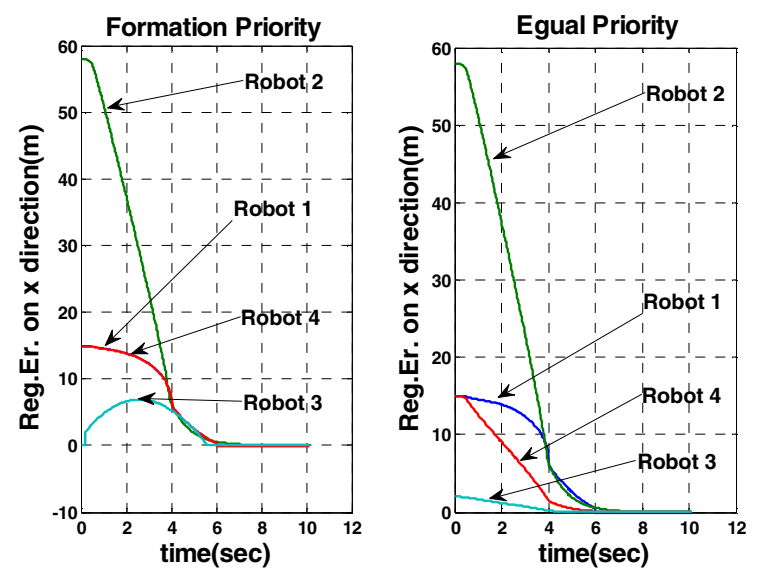

Fig. 3. Effects of value function on formation performance.

Fig. 3 illustrates the penalizing matrix effects on the formation performance of networked mobile robots. In Fig. 3 , the performance of the controller (29) with two different value functions are compared. The plot on the lefthand side depicts how the regulation errors converge when the penalizing matrix coefficients are chosen as $\chi_{1}=0.2 \chi_{2}$, and the second plot shows the regulation errors converge with the same penalizing matrices, $\chi_{1}=\chi_{2}$. As shown in Fig. 3, the third robot moves further away from its goal position at the beginning to reach consensus on regulation error, however, in the second plot, it converges to its goal position faster.

\section{CONCLUSIONS}

In this paper, a novel adaptive optimal consensus-based formation controller was derived for a network of mobile robots. The value function which was derived as a function of the regulation and formation errors was utilized to obtain optimal control inputs to each mobile robot such that the overall formation was able to move in consensus from any initial position to the desired target position. Simulation results confirm the theoretical claims.

\section{REFERENCES}

[1] R. Olfati-Saber, J. A. Fax, and R. M. Murray, "Consensus and cooperation in networked multi-agent systems," Proc. of IEEE, vol. 95, no. 2, pp. 215-233, 2007.

[2] S. Martinez, J. Cort'es, and F. Bullo, " Motion coordination with distributed information," IEEE Control Syst. Mag., vol. 2, no. 4, pp. 75-88, 2007.

[3] Y. Tian, and C. Liu, "Consensus of multi agent systems with diverse input and communication delays," IEEE Trans. Syst., Man, Cybern., Syst.-Part B, vol. 40, no. 2, pp. 362-370, 2010

[4] Y. Cao, Y. Li, W. Ren, and Y. Chen, "Distributed coordination of networked fractional-order systems," IEEE Trans. Syst., Man, Cybern., Syst., vol. 40, no. 2, pp. 362-370, 2010.

[5] D. Bauso, Le Giarre and R. Pesenti, "Consensus for networks with unknown but bounded disturbances," SIAM Journal of Control and Optimization, vol. 48, no. 3, pp. 1756-1770, 2009

[6] E. Semsar-Kazerooni and K. Khorasani, "Optimal consensus algorithms for cooperative team of agents subject to partial information," Automatica, vol. 44, no. 11, pp. 2766-2777, 2008.

[7] L. Sheng, Y.J. Pan, and X. Gong, "Consensus formation control for a class of networked multiple mobile robot systems," Journal of Control Science and Engineering, vol. 2012, 2012.

[8] H. M. Guzey and S. Jagannatan, "Adaptive neural network consensus based control of robot formations," Proc. SPIE vol. 8741, 87410M (2013)

[9] T. Dierks and S. Jagannathan, "Online optimal control of affine nonlinear discrete-time systems with unknown internal dynamics by using time-based policy update," IEEE Trans. Neural Netw. Learn. Syst., vol. 23, pp. 1118-1129, 2012.

[10] R. Fierro and F. L. Lewis, "Control of a nonholonomic mobile robot: Backstepping Kinematics into dynamics," Automation and Robotics Research Institute, The University of Texas at Arlington, 1996

[11] Q. Zhao, H. Xu and S. Jagannathan, " Adaptive dynamic programming-based state quantized networked control system without value and/or policy iterations," The 2012 International Joint Conference on Neural Networks (IJCNN), June 2012, pp.1-7.

[12] F. L. Lewis, S. Jagannathan, and A. Yesildirek, "Neural network control of robot manipulators and nonlinear systems", New York: Taylor \& Francis, 1999.

[13] T. Dierks, B. Brenner and S. Jagannathan, "Neural network-based optimal control of mobile robot formations with reduced information exchange," IEEE Trans. Control Syst. Technol., vol.21, no.4, pp.1407,1415, July 2013. 